\title{
Radiation unlikely to be responsible for high cancer rates among distal Hiroshima A-bomb survivors
}

\author{
Eric J. Grant · Yukiko Shimizu · Fumiyoshi Kasagi • \\ Harry M. Cullings $\cdot$ Roy E. Shore
}

Received: 4 March 2009/Accepted: 8 April 2009/Published online: 2 May 2009

(c) The Japanese Society for Hygiene 2009

\begin{abstract}
Watanabe et al. [1] (hereafter referred to as WMHY) used A-bomb survivor data from the Radiation Effects Research Foundation (RERF) Life Span Study (LSS) Report 12 (available online at http://www.rerf.or.jp) and mortality rates for Hiroshima and Okayama Prefectures [2] to create standardized mortality ratios (SMR) for LSS cohort members residing in Hiroshima at the time of the bombing. WMHY divided cohort members into three radiation dose categories: very low dose $(<0.005 \mathrm{~Gy}, \mathrm{VLD})$, low dose (0.005-0.1 Gy, LD), and high dose ( $>0.1 \mathrm{~Gy}, \mathrm{HD})$. At issue are the men in the VLD category, who experienced higher than expected deaths due to cancers compared with prefectural rates (note that, for brevity, we discuss only the solid cancer results). WMHY concluded that the increased cancer deaths among the VLD were due to underestimated neutron doses and/or unaccounted-for residual radiation exposures. We find their conclusions to be implausible and believe that the data show it is far more likely that the observed risks among the VLD are due to nonradiation factors. The reasons are briefly summarized below.
\end{abstract}

\footnotetext{
E. J. Grant $(\bowtie)$ · Y. Shimizu · F. Kasagi

Department of Epidemiology,

Radiation Effects Research Foundation,

5-2 Hijiyama Park, Minamiku,

Hiroshima 732-0815, Japan

e-mail: egrant@rerf.or.jp

H. M. Cullings

Department of Statistics, Radiation Effects Research Foundation,

5-2 Hijiyama Park, Minamiku, Hiroshima 732-0815, Japan

\section{R. E. Shore}

Directors' Office,

Radiation Effects Research Foundation,

5-2 Hijiyama Park, Minamiku,

Hiroshima 732-0815, Japan
}

\section{Implausible sex-specific risks}

If there were unaccounted-for radiation exposures, it is reasonable that they would have exposed men and women alike. However, the SMR values for VLD women were very close to 1.0; only those for men were elevated. In a related observation, previous studies have shown that the excess relative risk (ERR) per gray (Gy) of cancer mortality after radiation exposure is consistently higher for women compared with men [3, 4]. Thus, if radiation were responsible for the high SMR levels, we would expect to see higher SMRs in women compared with men. Using the estimates from Table 1 in WMHY [1], the sex ratio of excess risks for solid cancer is greater than unity in the HD group (female/male $=0.64 / 0.41=1.56$ ) as expected, but is less than unity for the $\operatorname{LD}$ group $(0.10 / 0.20=0.50)$ and even smaller for the VLD group $(0.04 / 0.18=0.22)$. This pattern supports the conclusion that the dominant cause for the high solid cancer SMR observed in the HD group is radiation, whereas, in the LD and VLD groups, the elevated SMRs are primarily due to nonradiation factors.

\section{Implausibly large effects}

If the elevated SMR value for the VLD male survivors was indeed due to radiation, the average acute dose level required to produce the observed SMR can be estimated by dividing the excess solid cancer mortality ratio for the VLD males (0.18) by the ERR/Gy estimate for males in the LSS cohort (0.38 [3]), giving $0.18 / 0.38 \mathrm{~Gy}^{-1} \approx 0.5 \mathrm{~Gy}$. This is a high level of exposure that was experienced by only about $7 \%$ of the LSS cohort, located at distances less than about $1,600 \mathrm{~m}$ from the hypocenter. Moreover, if a plume of radioactive materials were to have become airborne and 
returned to the ground several kilometers away (e.g., due to rain-out), the fallout doses would have to have been even higher than 0.5 Gy as only a fraction of the VLD would likely have been affected. We note that the Koi-Takusu area in the southwest part of Hiroshima has been identified as a fallout area but the levels of these exposures are estimated to be less than 0.05 Gy [5], far lower than would be necessary to produce the observed SMR value. It should also be noted that an ERR estimate similar to $0.38 \mathrm{~Gy}^{-1}$ is also supported by studies on other exposed cohorts around the world $[6,7]$.

\section{Other points}

WMHY suggest that underestimated initial doses and/or higher than assumed sensitivities to initial exposures could be responsible for the VLD group's high SMRs. Historical concerns regarding possible flaws in the DS86 dosimetry system (e.g., [8]) led to the development of a new dosimetry system (DS02) [9]. DS02 was developed by a panel of international experts and includes improvements in the source terms, radiation transport, and shielding assessments, and has been validated by the results of extensive physical measurements. However, the differences between the two dosimetry systems are quite modest, with the new system showing a general $\sim 10 \%$ increase in gamma-ray dose and a $\sim 10 \%$ decrease in neutron dose [10].

An argument based on higher sensitivities to low initial doses also seems to be unsupported as neither the RERF data [11], nor the weight of evidence from other radiation studies $[6,7]$, show higher risks per unit dose at low doses than at higher doses.

Finally, RERF researchers have previously reported evidence of heterogeneity in the mortality of proximal and distal members of the VLD $[12,13]$. WMHY attribute the higher SMR value to unaccounted-for radiation exposures. We believe a more plausible explanation, and one that is commonly observed in many studies, is that there are geographic variations in cancer mortality rates due to unmeasured factors (e.g., smoking).

\section{Conclusions}

We are gratified that another research group has availed themselves of these valuable data and published their interpretations. Such transparent exchange is a primary reason the data are made available. However, we see little support for WMHY's claims that the high SMR values observed in the VLD are due to unknown radiation exposures or high relative sensitivities to known low exposures. Rather, there is quantifiable evidence that refutes these claims, including the confirmed accuracy of the dosimetry system, external studies corroborating low-dose radiation sensitivities, observed effects far too large to be attributable to a plausible unknown radiation dose, and cancer risk sex ratio patterns inconsistent with those known to occur after radiation exposure.

We agree that the data that RERF have collected and refined on dosimetry and risk factors for disease may only partially reflect the unique risks of the A-bomb survivors. Nevertheless, we have extensive data describing the patterns in which radiation effects appear and the general magnitude of those effects. We do not believe that the radiation-based arguments presented by WMHY to explain the high SMR values in the VLD are compatible with either the health effects evidence observed in the LSS or what is known about radiation effects in humans from other radiation studies [6].

The authors are employed by RERF but this commentary does not constitute an official statement of the position of RERF or its funding agencies. RERF is a private, nonprofit foundation funded equally by the Japanese Ministry of Health, Labour, and Welfare, and the US Department of Energy, the latter in part through the US National Academy of Sciences. RERF's mission is strictly scientific in nature.

\section{References}

1. Watanabe T, Miyao M, Honda R, Yamada Y. Hiroshima survivors exposed to very low doses of A-bomb primary radiation showed a high risk for cancers. Environ Health Prev Med. 2008;13:264-70.

2. Age-adjusted death rates by prefecture. Special report on vital statistics. Japan:Statistics and Information Department, Minister's Secretariat, Ministry of Health and Welfare; 1975, 1980, 1985, 1990.

3. Pierce DA, Shimizu Y, Preston DL, Vaeth M, Mabuchi K. Studies of the mortality of atomic bomb survivors. Report 12, Part I. Cancer: 1950-1990. Radiat Res. 1996;146:1-27.

4. Preston DL, Pierce DA, Shimizu Y, Cullings HM, Fujita S, Funamoto $S$, et al. Effect of recent changes in atomic bomb survivor dosimetry on cancer mortality risk estimates. Radiat Res. 2004;162:377-89.

5. Roesch WC (ed.) US-Japan joint reassessment of atomic bomb radiation dosimetry in Hiroshima and Nagasaki, final report. Hiroshima, Japan: radiation effects research foundation; 1987.

6. Effects of ionizing radiation : UNSCEAR 2006 report to the General Assembly, with scientific annexes/United Nations Scientific Committee on the Effects of Atomic Radiation (UNSCEAR). New York: United Nations; 2008.

7. National Research Council, Committee to Assess Health Risks from Exposure to Low Levels of Ionizing Radiation (BEIR VII). Washington: National Academies Press; 2005.

8. Straume T, Egbert S, Woolson W, Finkel R, Kubik P, Grove H, et al. Neutron discrepancies in the DS86 Hiroshima dosimetry system. Health Phys. 1992;63:421-6.

9. Young RW, Kerr GD, editors. Reassessment of the atomic bomb radiation dosimetry for Hiroshima and Nagasaki-dosimetry 
system 2002. Hiroshima: Radiation Effects Research Foundation; 2005.

10. Cullings HM, Levenson Z, Funamoto S, Teranishi S. Changes in atomic bomb survivors' dosimetry with the new dosimetry system DS02. Jpn J Health Phys. 2006;41:161-271.

11. Ban S, Cologne JB, Fujita S, Awa AA. Radiosensitivity of atomic bomb survivors as determined with a micronucleus assay. Radiat Res. 1993;134:170-8.
12. Cologne JB, Preston DL. Longevity of atomic-bomb survivors. Lancet. 2000;356:303-7.

13. Cologne JB, Preston DL. Impact of comparison group on cohort dose response regression: an example using risk estimation in atomic-bomb survivors. Health Phys. 2001;80:491-6. 